\title{
Trunk appearance perception scale (TAPS) discrepancy between adolescents with idiopathic scoliosis and their parents influences HRQL
}

\author{
M Rigo ${ }^{* *}$, E D'Agata $^{2}$, M Jelacic ${ }^{1}$ \\ From 9th International Conference on Conservative Management of Spinal Deformities - SOSORT 2012 \\ Annual Meeting \\ Milan, Italy. 10-12 May 2012
}

\section{Background}

The Trunk Appearance Perception Scale (TAPS) is a valid instrument to assess self-perception of trunk deformity [1]. The SRS-22 has been widely used to measure Health Related Quality of Life in scoliosis population, but it is not clear which factors can influence its final score [2]. Children and parents can perceive trunk deformity differently, and discrepancy can be assessed with a coefficient of discrepancy $(\mathrm{CD}=$ TAPS major - TAPS minor $\mathrm{x} 100 /$ TAPS major). We already found that the CD influences the SRS-22 score. However, the previous study included patients older than 18 years, both genders, and results were not reported according to age [3].

\section{Aim}

The aim of this new study is to confirm previous results, in a larger sample size, including only girls from 10 to 18 years of age (adolescents).

\section{Methods}

Prospective study including 107 girls diagnosed with idiopathic scoliosis (treated and untreated), attending the clinic with their parents. Mean age 14.4 years. Mean Cobb angle $33.5^{\circ}$ (10-75). All patients completed the SRS-22 and the TAPS. Parents completed the TAPS assessing trunk deformity of their children. A coefficient of discrepancy (TAPS-CD) was defined. Statistical analysis (SPSS) was made to compare TAPS, TAPS-CD and SRS-22.

\section{Results}

Results confirmed previous findings. A significant correlation was found between patients' TAPS and SRS-subtotal,

${ }^{1}$ Institut Elena Salvà, Barcelona, Spain

Full list of author information is available at the end of the article pain, self-image and mental health and between parents' TAPS, function and treatment satisfaction in the SRS-22. Parents' TAPS did not correlate with the Cobb angle, but with SRS-subtotal of the girls' self-image, pain and mental health. TAPS CD showed a significant correlation with SRS-Subtotal, self-image and mental health. Two groups were created according to the SRS-22 score. Patients with lower score in the SRS-22 showed a higher TAPS-CD $(\mathrm{P}<.05)$. These results were not different in girls aged 10 to 14 and girls aged 14 to 18 .

\section{Conclusion}

Discrepancy in "perception of trunk deformity" between adolescent girls and parents influence the SRS-22. Whether such a discrepancy is a factor for a lower quality of life, later, during adult life, is an open question.

\section{Author details \\ ${ }^{1}$ Institut Elena Salvà, Barcelona, Spain. ${ }^{2}$ Fund. Hosp. Univers. Vall D'Hebron - Institut de Recerca. Barcelona, Spain.}

Published: 3 June 2013

\section{References}

1. Bago J, Sanchez-Raya J, Perez-Grueso FJ, Climent JM: The Trunk Appearance Perception Scale (TAPS): a new tool to evaluate subjective impression of trunk deformity in patients with idiopathic scoliosis. Scoliosis 2010, 5:6.

2. Climent JM, Bago J, Ey A, Perez-Grueso FJ, Izquierdo E: Validity of the Spanish version of the Scoliosis Research Society-22 (SRS-22) Patient Questionnaire. Spine 2005, 30(6):705-709.

3. Rigo M, DA E, Jelacic E: Trunk appearance perception scale (TAPS) discrepancy between scoliosis children and their parents influences the SRS-22 score. scoliosis 2012, 7(suppl 1):03.

doi:10.1186/1748-7161-8-S1-055

Cite this article as: Rigo et al.: Trunk appearance perception scale (TAPS) discrepancy between adolescents with idiopathic scoliosis and their parents influences HRQL. Scoliosis 2013 8(Suppl 1):055. 